\title{
Revisión de estudios de coste- efectividad en servicios de atención comunitaria y atención centrada en la persona
}

\section{Madalen Saizarbitoria}

SIIS Centro de Documentación y Estudios Fundación Eguía-Careaga

<estudios@siis.net>

SIIS Dokumentazio eta Ikerketa Zentroak Matia Fundazioarentzat eginiko berrikusketa honetan, pertsonan zentratutako eta arreta komunitarioko esku-hartze eta programen eraginkortasuna eta kostuaren arteko lotura aztertzen da.

\section{HITZ-GAKOAK:}

kostua, eraginkortasuna, berrikusketa, zientzialiteratura, arreta soziosanitarioa.
En esta revisión elaborada por el SIIS Centro de Documentación y Estudios para la Fundación Matia, se analiza la relación coste-efectividad de las intervenciones y programas comunitarios y centrados en la persona.

\section{Palabras Clave:}

coste, efectividad, revisión, literatura científica, atención sociosanitaria. 


\section{Introducción}

La Fundación Matia ha puesto en práctica un proceso de investigación para el diseño de nuevas formas de cuidado a personas dependientes o con discapacidad, con el objeto de desarrollar un nuevo modelo de atención comunitaria, individualizada e integral, en el ámbito del espacio sociosanitario. En el marco de ese proceso, se ha considerado necesario valorar los resultados que, en términos de coste-efectividad, supondría la adopción de un modelo de esas características, a partir de la literatura científica existente al respecto en los países de nuestro entorno.

Para ello, se ha solicitado al SIIS Centro de Documentación y Estudios de la Fundación Eguía-Careaga que elabore un estudio de revisión de la literatura científica que analiza el impacto económico y la relación coste-efectividad de las intervenciones, los programas o las políticas basadas en enfoques coincidentes con los del modelo que la Fundación Matia propone desarrollar. El objetivo final de este análisis es el de conocer la evidencia científica disponible en cuanto a la relación coste-efectividad de intervenciones y políticas de atención que puedan asociarse al paradigma de intervención planteado por la Fundación Matia, de forma que, a la hora de plantear el contenido de ese modelo, puedan incorporarse a él todas aquellas intervenciones, prácticas o enfoques que hayan demostrado ya, mediante estudios de evaluación rigurosos y de contrastada calidad, una relación positiva de coste-efectividad.

La revisión que se describe en este informe es una revisión sistemática cuyo objetivo consiste en sintetizar el conocimiento científico actualmente existente sobre las intervenciones que mejores resultados obtienen en el ámbito de la atención a personas mayores, tanto en términos de la calidad de la atención dispensada, como de su coste. Esto permitirá a las personas responsables de los proyectos de la Fundación Matia adoptar sus decisiones basándose en la mejor evidencia disponible y, por lo tanto, avanzar por el camino de la práctica basada en la evidencia.

De acuerdo con los objetivos del trabajo, se ha procedido a realizar una búsqueda sistemática de estudios científicos publicados sobre la relación coste-efectividad de intervenciones sociales o sociosanitarias para personas mayores o personas con discapacidad. Las búsquedas se han realizado siguiendo un protocolo que implica la progresión desde bases de datos más especializadas a aquellas de corte más general. En concreto, la búsqueda se ha realizado en cinco bases de datos: la base de datos bibliográfica del SIIS Centro de Documentación y Estudios, las bases de datos de revisiones sistemáticas y de evaluaciones económicas del Centre for Reviews and Dissemination (CRD) del Sistema Nacional de Salud (NHS) británico, la Biblioteca Cochrane, Pubmed y la base de datos científica Web of Knowledge (WOK).

Los criterios de búsqueda utilizados han sido los siguientes:
- Personas mayores o personas con discapacidad o personas con enfermedades crónicas o personas con enfermedades terminales o personas con dependencia o demencia o Alzheimer o personas con enfermedad mental; $y$

- atención social o servicios sociales o servicios sociosanitarios; y

- coordinación sociosanitaria o espacio sociosanitario o atención integrada o intervención comunitaria o servicios comunitarios o atención comunitaria o planificación centrada en la persona 0 atención centrada en la persona 0 individualización de la atención o personalización de la atención; y

- coste-eficacia o coste-utilidad o coste-efectividad o coste-beneficio.

Tras aplicar estos criterios, se han excluido 142 estudios, reduciéndose la selección final a 84 documentos, que constituyen la muestra finalmente analizada. Hay que tener en cuenta, no obstante, que 28 de los 84 documentos revisados son, en realidad, revisiones de la literatura que, por lo tanto, son, a su vez, una síntesis de cientos de estudios de investigación. Aunque algunos de los estudios individuales incluidos en esta revisión ya habían sido considerados en alguna de las revisiones utilizadas, esto amplía considerablemente el alcance de este trabajo ${ }^{1}$.

Los estudios incluidos se han clasificado en siete tipos, de acuerdo con su diseño metodológico. Por orden descendiente de calidad en relación con la fiabilidad de los datos que pueden aportar, se distinguen: las revisiones de la literatura científica, los ensayos aleatorios o estudios controlados aleatorios, los estudios controlados no aleatorios, los estudios pre-post sin grupo de control, los estudios exploratorios, los estudios de casos y los estudios teóricos.

\section{Resultados de la revisión de los estudios}

\subsection{Descripción}

\subsubsection{Tipo de estudio y calidad metodológica}

Se han encontrado y revisado 84 artículos e informes en los que se analiza la eficiencia de servicios e intervenciones coincidentes con el modelo de atención objeto de estudio en términos de su coste y efectividad. Prácticamente una cuarta parte de esos estudios (20 de los 84) son revisiones de la literatura que sintetizan los resultados de evaluaciones económicas anteriores; la tercera parte (28 de los 84 ) son ensayos aleatorios que, en principio, pueden poseer una buena validez interna y, por lo tanto, ofrecen mayor certeza de que existe una relación causal entre la intervención realizada y el efecto observado; y algo más de una quinta parte son estudios con grupo de

${ }^{1}$ De hecho, constituye una buena práctica en la realización de revisiones de la literatura considerar en primer lugar revisiones realizadas con anterioridad. 
control. Tal y como se ha explicado anteriormente, también se han incluido algunos estudios con metodologías menos robustas, como estudios pre-post sin grupo de control (cinco documentos), estudios exploratorios (siete documentos) o estudios de casos (dos documentos). Finalmente, cuatro documentos estiman el impacto de las intervenciones que evalúan de forma teórica.

Tabla 1. Estudios revisados, por tipo de diseño utilizado

\begin{tabular}{l|c|c}
\hline Tipo de diseño & $\begin{array}{c}\text { № de } \\
\text { documentos }\end{array}$ & $\%$ \\
\hline Revisión de la literatura & 20 & 23,8 \\
\hline Ensayo aleatorio & 28 & 33,3 \\
\hline Estudio controlado no aleatorio & 18 & 21,4 \\
\hline Estudio pre-post sin grupo control & 5 & 6,0 \\
\hline Estudio de casos & 2 & 2,4 \\
\hline Estudio exploratorio & 7 & 8,3 \\
\hline Modelo teórico & 4 & 4,8 \\
\hline Total & $\mathbf{8 4}$ & $\mathbf{1 0 0 , 0}$ \\
\hline
\end{tabular}

Fuente: Elaboración propia.

Atendiendo al tipo de evaluación económica realizada, 50 de los 84 analizan la relación coste-efectividad de la intervención, doce son estudios de costeutilidad (traducen todos los beneficios a un mismo indicador), y cuatro son estudios de coste-beneficio (traducen los beneficios a términos económicos).

Tabla 2. Estudios revisados, por tipo de evaluación económica de que se trate

\begin{tabular}{l|c|c}
\hline Tipo de evaluación económica & $\begin{array}{c}\text { No de } \\
\text { documentos }\end{array}$ & $\%$ \\
\hline Estudio de coste-eficacia & 51 & 60,7 \\
\hline Estudio de coste-utilidad & 9 & 10,7 \\
\hline Estudio de coste-beneficio & 4 & 4,8 \\
\hline Estudio de minimización de costes & 2 & 2,4 \\
\hline Estudio de costes & 7 & 8,3 \\
\hline No es una evaluación económica completa & 4 & 4,8 \\
\hline No se puede determinar & 7 & 8,3 \\
\hline Total & $\mathbf{8 4}$ & $\mathbf{1 0 0 , 0}$ \\
\hline
\end{tabular}

Fuente: Elaboración propia.

De los 60 estudios revisados que evalúan una intervención de forma experimental (excluyendo, por tanto, las revisiones de la literatura y los modelos teóricos), una tercera parte provienen del Reino Unido y prácticamente otros tantos de los Estados Unidos. El resto provienen de países como Canadá, Suecia, España, Países Bajos, Irlanda, Italia, Australia, China, Francia, Noruega y Nueva Zelanda. Estas cifras no sólo reflejan la mayor tradición investigadora en el ámbito de la intervención social en los países anglosajones, sino también, y de forma significativa, la mayor preocupación que existe en esos países por la eficiencia y la productividad de las intervenciones sociosanitarias.

Por lo que se refiere al año de publicación de los estudios, dado que a la hora de realizar la búsqueda de documentos la selección no se ha restringido a los publicados con posterioridad a una fecha, existe una gran variedad, desde los más antiguos, que se remontan a finales de la década de los ochenta, hasta los más recientes, publicados en 2011. En cualquier caso, la mayoría de los estudios incluidos ( 44 de los 84 revisados) son posteriores a 2005 y una tercera parte (26 de los 84) se publicaron entre 2000 y 2005. Esta distribución refleja probablemente un creciente interés por la relación coste-efectividad de las intervenciones sociales en el ámbito internacional, así como un progresivo desarrollo de las técnicas y las metodologías necesarias para la aplicación práctica de este tipo de enfoques.

En general, y teniendo presente que, tal y como se ha mencionado, la escala utilizada para la selección de los documentos es muy rigurosa, dadas las condiciones en las que frecuentemente tiene que llevarse a cabo la investigación en el ámbito sociosanitario, la validez interna de los estudios incluidos en la revisión puede calificarse de media-baja. De los 56 estudios que se han podido valorar (19 estudios eran revisiones sistemáticas y 9 documentos no daban información suficiente para aplicar el instrumento de valoración); 7 tienen una buena validez interna, es decir, se trata de ensayos aleatorios con un buen diseño; y 26 tienen una calidad aceptable, es decir, son ensayos aleatorios de calidad media o estudios controlados no aleatorios con un adecuado control de los posibles factores de confusión. Los 22 estudios restantes son pobres en cuanto a su validez interna, es decir, no controlan adecuadamente los posibles factores de confusión, lo que, si bien no invalida sus conclusiones, sí introduce un cierto factor de incertidumbre en ellas.

Tabla 3. Estudios revisados, según su validez interna

\begin{tabular}{l|c|c|c}
\hline Validez interna del estudio & $\begin{array}{c}\text { № de } \\
\text { documentos }\end{array}$ & $\%$ & \% válido \\
\hline Excelente & 1 & 1,2 & 1,8 \\
\hline Buena & 7 & 8,3 & 12,5 \\
\hline Satisfactoria & 26 & 31,0 & 46,4 \\
\hline Pobre & 22 & 26,2 & 39,3 \\
\hline $\begin{array}{l}\text { No aplicable (revisiones } \\
\text { sistemáticas/información } \\
\text { insuficiente) }\end{array}$ & 28 & 33,3 & 100,0 \\
\hline \begin{tabular}{l} 
Total \\
\hline
\end{tabular} & $\mathbf{8 4}$ & $\mathbf{1 0 0 , 0}$ & - \\
\hline
\end{tabular}

Fuente: Elaboración propia.

Por el contrario, puede decirse que, en general, la validez externa de los estudios analizados es mucho mejor, pudiéndose considerar los resultados adecuadamente extrapolables a toda la población objeto de estudio en 
28 de los 57 estudios valorados, y de generalizabilidad limitada solamente en 8 casos. En 28 de los casos, en la medida en que se trata de revisiones de otros estudios, el criterio de validez externa no se aplica.

Tabla 4. Estudios revisados, según su validez externa

\begin{tabular}{l|c|c|c}
\hline Validez externa del estudio & $\begin{array}{c}\text { № de } \\
\text { documentos }\end{array}$ & $\%$ & \% válido \\
\hline Buena & 47 & 56,0 & 83,9 \\
\hline Limitada & 7 & 8,3 & 12,5 \\
\hline Pobre & 2 & 2,4 & 3,6 \\
\hline No aplicable & 28 & 33,3 & 100,0 \\
\hline Total & $\mathbf{8 4}$ & $\mathbf{1 0 0 , 0}$ & - \\
\hline
\end{tabular}

Fuente: Elaboración propia.

Finalmente, por lo que se refiere a la calidad de la evaluación económica, la mayoría de los estudios revisados tienen una calidad buena (43,6\%) o aceptable (43,6\%), de acuerdo con los criterios señalados anteriormente.

Tabla 5. Estudios revisados, por calidad de la evaluación económica

\begin{tabular}{l|c|c|c}
\hline $\begin{array}{l}\text { Calidad de la evaluación } \\
\text { económica }\end{array}$ & $\begin{array}{c}\text { № de } \\
\text { documentos }\end{array}$ & $\%$ & \% válido \\
\hline Buena & 24 & 28,6 & 43,6 \\
\hline Aceptable & 24 & 28,6 & 43,6 \\
\hline Pobre & 7 & 8,3 & 12,7 \\
\hline No se puede determinar & 1 & 1,2 & 100,0 \\
\hline $\begin{array}{l}\text { No aplicable (revisiones } \\
\text { sistemáticas/información } \\
\text { insuficiente) }\end{array}$ & 28 & 33,3 & - \\
\hline \begin{tabular}{l} 
Total \\
\hline
\end{tabular} & $\mathbf{8 4}$ & $\mathbf{1 0 0 , 0}$ & - \\
\hline
\end{tabular}

Fuente: Elaboración propia.

\subsubsection{Tipo de intervenciones evaluadas}

Los 84 documentos revisados analizan un total de 92 intervenciones distintas, que se han agrupado en 18 grandes grupos. En la mayoría de los casos, se trata de fórmulas de alojamiento en la comunidad (15 documentos), fórmulas de coordinación de servicios sociosanitarios (11 documentos), intervenciones de promoción del autocuidado (10 documentos), intervenciones de gestión de casos (8 documentos), servicios de día, ayudas tecnológicas y programas de prevención de caídas (6 documentos), o fórmulas de individualización de la atención (5 documentos). Para otras intervenciones que pueden ser de especial interés en el marco de este trabajo, como la planificación centrada en la persona, se han encontrado muy pocos estudios que incorporen una evaluación económica.

Casi la mitad de los documentos que se han incluido en la revisión se centran en cuatro tipos de intervenciones específicas: las fórmulas de alojamiento en la comunidad, la coordinación entre los servicios sociales y sanitarios en el ámbito del espacio sociosa- nitario, el autocuidado y la gestión de casos. La propia relevancia de estos tipos de intervención en el contexto de la literatura analizada pone ya de manifiesto, al margen de los resultados obtenidos en términos de coste-efectividad, que son éstas las intervenciones que en mayor medida se han puesto en práctica, y se han evaluado, en los países de nuestro entorno.

Por lo que se refiere a los colectivos destinatarios de las intervenciones evaluadas, 59 de las 92 se refieren a personas mayores, ya sean personas mayores dependientes o en riesgo de dependencia. También se han incluido 18 intervenciones dirigidas a personas con discapacidad, 3 a personas con enfermedades crónicas y 12 dirigidas a personas que se encargan del cuidado informal de personas mayores o con discapacidad.

Las 92 intervenciones evaluadas refieren un total de 185 objetivos diferentes, que han sido agrupados en trece objetivos básicos. El propio listado resulta de interés, en la medida en que pone de manifiesto los objetivos fundamentales que se buscan mediante la puesta en práctica de las intervenciones analizadas. De acuerdo con la Tabla 6, cuatro objetivos concentran algo más de la mitad de todas las intervenciones analizadas: la mejora de la calidad de vida de las personas usuarias, señalado en 31 ocasiones; la reducción en el uso de servicios sanitarios, en 25 ocasiones; el retraso o la evitación de la institucionalización, en 23 ocasiones; y la mejora del estado de salud de las personas objeto de la intervención, en 18 ocasiones. Además, 19 intervenciones tienen el objetivo añadido de reducir el coste de la atención prestada.

Tabla 6. Intervenciones evaluadas, por tipos de objetivos perseguidos

\begin{tabular}{|c|c|c|}
\hline Objetivo de la intervención & $\begin{array}{c}\text { № de } \\
\text { intervenciones }\end{array}$ & $\%$ \\
\hline Mejorar la calidad de vida & 31 & 16,8 \\
\hline Reducir el uso de servicios sanitarios & 25 & 13,5 \\
\hline Retrasar o evitar la institucionalización & 23 & 12,4 \\
\hline Reducir el coste & 19 & 10,3 \\
\hline $\begin{array}{l}\text { Mejorar el estado de salud de las personas } \\
\text { usuarias }\end{array}$ & 18 & 9,7 \\
\hline $\begin{array}{l}\text { Incrementar el grado de satisfacción de las } \\
\text { personas usuarias }\end{array}$ & 16 & 8,6 \\
\hline Mejorar o mantener la capacidad funcional & 14 & 7,6 \\
\hline $\begin{array}{l}\text { Reducir la carga de trabajo de las personas } \\
\text { cuidadoras }\end{array}$ & 11 & 5,9 \\
\hline Reducir el uso de servicios formales & 11 & 5,9 \\
\hline $\begin{array}{l}\text { Incrementar la autodeterminación o el } \\
\text { autocontrol de las personas usuarias }\end{array}$ & 7 & 3,8 \\
\hline Prevención de caídas & 6 & 3,2 \\
\hline $\begin{array}{l}\text { Empoderamiento / incrementar la } \\
\text { autoeficacia }\end{array}$ & 3 & 1,6 \\
\hline $\begin{array}{l}\text { Incrementar el acceso a servicios } \\
\text { comunitarios preventivos }\end{array}$ & 1 & 0,5 \\
\hline Total & 185 & 100,0 \\
\hline
\end{tabular}

Fuente: Elaboración propia. 


\subsection{Resultados sobre coste-efectividad}

Cincuenta y ocho de las noventa y dos intervenciones evaluadas pueden considerarse coste-efectivas, mientras que cuatro no pueden ser consideradas como tales. En catorce de los casos analizados se obtienen mejores resultados a un mayor coste, o peores resultados a un menor coste, con lo que habría que valorar si el incremento o la reducción del coste compensan la mejora o la reducción de la efectividad. Finalmente, para dieciséis de las intervenciones evaluadas no existe información suficiente para afirmar si la intervención resulta o no coste-efectiva, o los resultados son contradictorios.

Si el porcentaje de intervenciones consideradas coste-efectivas se analiza desde el punto de vista de los colectivos destinatarios de la atención, se observa -dejando al margen a las personas con enfermedades crónicas, a quienes se dirigen solamente tres de las intervenciones analizadasque es en el caso de las intervenciones dirigidas a personas con discapacidad donde se registra un mayor porcentaje de intervenciones coste-efectivas, puesto que prácticamente dos de cada tres intervenciones analizadas se han considerado como tales. En el caso de las personas mayores, se consideraron coste-efectivas el 63,9\% de las intervenciones, y en el caso de las intervenciones destinadas a las personas cuidadoras informales, únicamente el $40 \%$. En este caso, cuatro de cada diez estudios se consideraron no concluyentes.

Tabla 7. Intervenciones evaluadas, por valoración sobre coste-efectividad y colectivo destinatario de la atención

\begin{tabular}{l|c|c|c}
\hline \multirow{2}{*}{ Colectivo } & Resultados & $\begin{array}{c}\text { № de } \\
\text { intervenciones }\end{array}$ & $\%$ \\
\hline \multirow{4}{*}{$\begin{array}{l}\text { Personas } \\
\text { mayores }\end{array}$} & Coste-efectivo & 39 & 63,9 \\
\cline { 2 - 4 } & No coste-efectivo & 2 & 3,3 \\
\cline { 2 - 4 } & No dominante & 9 & 14,8 \\
\cline { 2 - 4 } & No concluyente & 9 & 3,3 \\
\hline \multirow{3}{*}{$\begin{array}{l}\text { Personas con } \\
\text { discapacidad }\end{array}$} & No dominante & 3 & 14,8 \\
\cline { 2 - 4 } & No concluyente & 3 & 16,7 \\
\hline \multirow{3}{*}{$\begin{array}{l}\text { Cuidadores } \\
\text { informales }\end{array}$} & Coste-efectivo & 4 & 40,0 \\
\cline { 2 - 4 } & No coste-efectivo & 2 & 20,0 \\
\cline { 2 - 4 } & No concluyente & 4 & 40,0 \\
\hline $\begin{array}{l}\text { Personas con } \\
\text { enfermedades } \\
\text { crónicas }\end{array}$ & Coste-efectivo & 3 & 100,0 \\
\hline
\end{tabular}

Fuente: Elaboración propia.

No puede decirse, por otra parte, que el tipo de metodología utilizada en la revisión se relacione con el porcentaje de intervenciones consideradas costeefectivas, y menos aún que las intervenciones realizadas con los métodos a priori más exigentes, los estudios controlados aleatorios, registren un porcentaje de intervenciones coste-efectivas más bajo. Al contrario, los estudios controlados aleatorios resultan coste-efectivos en el $67,9 \%$ de los casos, y los estudios controlados no aleatorios, en el 66,7\%. Ambos tipos de estudios se encuentran, de hecho, entre los que registran un porcentaje más elevado de resultados coste-efectivos.

Tabla 8. Intervenciones evaluadas, por valoración sobre coste-efectividad y metodología utilizada

\begin{tabular}{|c|c|c|c|}
\hline Tipo de estudio & Resultado & N & $\%$ \\
\hline \multirow{5}{*}{$\begin{array}{l}\text { Estudio controlado } \\
\text { aleatorio }\end{array}$} & Coste-efectivo & 19 & 67,9 \\
\hline & No coste-efectivo & 2 & 7,1 \\
\hline & No dominante & 3 & 10,7 \\
\hline & Neutro & 2 & 7,1 \\
\hline & No concluyente & 2 & 7,1 \\
\hline \multirow{3}{*}{$\begin{array}{l}\text { Estudio controlado no } \\
\text { aleatorio }\end{array}$} & Coste-efectivo & 12 & 66,7 \\
\hline & No dominante & 3 & 16,7 \\
\hline & No concluyente & 3 & 16,7 \\
\hline \multirow{3}{*}{$\begin{array}{l}\text { Estudio pre-post sin } \\
\text { grupo control }\end{array}$} & Coste-efectivo & 1 & 20,0 \\
\hline & No dominante & 3 & 60,0 \\
\hline & No concluyente & 1 & 20,0 \\
\hline \multirow{3}{*}{ Estudio exploratorio } & Coste-efectivo & 5 & 71,4 \\
\hline & No dominante & 1 & 14,3 \\
\hline & No concluyente & 1 & 14,3 \\
\hline \multirow{2}{*}{ Estudio de casos } & Coste-efectivo & 1 & 50,0 \\
\hline & No concluyente & 1 & 50,0 \\
\hline Estudio teórico & Coste-efectivo & 4 & 100,0 \\
\hline \multirow{4}{*}{ Revisión de la literatura } & Coste-efectivo & 16 & 57,1 \\
\hline & No coste-efectivo & 2 & 7,1 \\
\hline & No dominante & 2 & 7,1 \\
\hline & No concluyente & 8 & 28,6 \\
\hline
\end{tabular}

Fuente: Elaboración propia.

Desde el punto de vista del tipo de intervención, las que en mayor medida se han considerado costeefectivas son las relacionadas con la coordinación de servicios sociosanitarios, ya sea a través de la integración (100\% de intervenciones coste-efectivas), o a través de la gestión de casos $(87,5 \%$ de costeefectividad). Le siguen los programas orientados a la prestación de ayudas tecnológicas ( $83 \%$ de intervenciones consideradas coste-efectivas), los programas de promoción del autocuidado (80\%, con diez intervenciones analizadas), las visitas domiciliarias preventivas $(75 \%)$ y las fórmulas de alojamiento en la comunidad ( $73 \%$, con quince intervenciones analizadas). También registran un porcentaje elevado de intervenciones consideradas coste-efectivas los servicios de atención domiciliaria (67\%), si bien en estos casos el número de intervenciones evaluadas es más reducido. 
En general, las intervenciones menos evaluadas son las que en mayor medida muestran resultados no concluyentes, no dominantes o no coste-efectivos. Así por ejemplo, ninguna de las dos intervenciones orientadas a los servicios de rehabilitación se ha mostrado coste-efectiva, y sólo una de las tres intervenciones centradas en los servicios de respiro o en los programas de asignación económica individual.

Tabla 9. Intervenciones coste-efectivas por tipo de intervención

\begin{tabular}{|c|c|c|}
\hline Tipo de intervención & $\begin{array}{c}\text { № de } \\
\text { intervenciones } \\
\text { coste-efectivas }\end{array}$ & $\begin{array}{c}\% \text { sobre el } \\
\text { total de cada } \\
\text { tipo }\end{array}$ \\
\hline Servicios sociosanitarios integrados & 4 & 100,0 \\
\hline Gestión de casos & 7 & 87,5 \\
\hline Ayudas tecnológicas & 5 & 83,3 \\
\hline Autocuidado & 8 & 80,0 \\
\hline Visitas domiciliarias preventivas & 3 & 75,0 \\
\hline $\begin{array}{l}\text { Fórmulas de alojamiento en la } \\
\text { comunidad }\end{array}$ & 11 & 73,3 \\
\hline Ayuda a domicilio & 2 & 66,7 \\
\hline $\begin{array}{l}\text { Otras fórmulas de individualización } \\
\text { de la atención }\end{array}$ & 3 & 60,0 \\
\hline $\begin{array}{l}\text { Otras fórmulas de coordinación de } \\
\text { servicios sociosanitarios }\end{array}$ & 6 & 54,5 \\
\hline Servicios comunitarios en general & 1 & 50,0 \\
\hline Servicios de día & 3 & 50,0 \\
\hline Prevención de caídas & 3 & 50,0 \\
\hline Servicios de respiro & 1 & 33,3 \\
\hline Asignación económica individual & 1 & 33,3 \\
\hline Servicios de rehabilitación & 0 & 0,0 \\
\hline Re-capacitación a domicilio & 0 & 0,0 \\
\hline Servicios de acompañamiento & 0 & 0,0 \\
\hline Planificación centrada en la persona & 0 & 0,0 \\
\hline
\end{tabular}

Fuente: Elaboración propia.

Hay también determinados tipos de intervención que no parecen mostrarse en ningún caso costeefectivos: se trata de los servicios de rehabilitación, los de re-capacitación a domicilio, los servicios de acompañamiento y los de planificación centrada en la persona. Se trata, en cualquier caso, de intervenciones sometidas a muy escasas evaluaciones (en general, una sola) que tienden a mostrar resultados no concluyentes o no dominantes, es decir, a obtener resultados positivos en términos de efectividad, si bien a un coste más elevado que las intervenciones alternativas con las que se comparan.

Los resultados de la Tabla 9 han de ser interpretados con precaución: no puede derivarse de ellos, por ejemplo, que los programas de gestión de casos sean en todo momento coste-efectivos, o que cualquier programa de gestión de casos que se desarrolle lo vaya a ser. En ese sentido, hay que tener en cuenta al menos tres cuestiones:

- Por una parte, no debe olvidarse que el carácter coste-efectivo de una intervención se establece comparándola con una intervención alternativa. Cabría la posibilidad de que, de aplicarse en otro contexto, y al compararse con otra intervención, un mismo programa dejara de considerarse coste-efectiva.

- Por otra parte, el carácter coste-efectivo de un programa no implica que, incluso aunque se aplicara de la misma forma, fuera a resultar igualmente coste-efectivo en otro contexto o circunstancia.

- Por último, el hecho de que una serie de intervenciones determinadas -por ejemplo, las relacionadas con la gestión de casos- hayan sido consideradas mayoritariamente coste-efectivas no implica que todas las intervenciones realizadas en esa misma línea lo vayan a ser. Ello dependerá, lógicamente, de la forma concreta en la que se materialice esa intervención, del perfil de las personas atenidas o de los recursos invertidos.

Los datos de la tabla sí indican, sin embargo, que existen una serie de ámbitos de intervención fundamentalmente, la gestión de casos, las ayudas tecnológicas, los programas de autocuidado, los servicios sociosanitarios integrados, las fórmulas de alojamiento en la comunidad y los servicios de ayuda a domicilio- que se han evaluado en un número considerable de ocasiones y que han resultado coste-efectivas en un porcentaje muy elevado de esas ocasiones. Puede decirse, por tanto, que se trata de líneas de intervención con un elevado potencial en términos de coste-efectividad, en la medida en que, en la mayoría de los casos en los que se han desarrollado y se han evaluado con criterios metodológicos rigurosos y contrastados, se ha hallado una relación de coste-efectividad positiva.

\section{Principales conclusiones}

Las conclusiones de este informe constituyen una base sólida sobre la que ir construyendo un modelo de atención a personas mayores que no sólo sea más efectivo en términos de mejora de su grado de autonomía, su participación y empoderamiento, su grado de satisfacción con los servicios recibidos 0 , en definitiva, su calidad de vida, sino que, además, alcance esos objetivos de la manera más eficiente posible desde el punto de vista económico, permitiendo utilizar los recursos allí donde más útiles pueden resultar.

La solidez de las conclusiones se deriva fundamentalmente del elevado número de investigaciones analizadas y del cumplimiento de una serie de criterios temáticos y metodológicos estrictos para su inclusión en este estudio. Aun teniendo en cuenta las limitaciones señaladas en el punto anterior, la revisión 
de la literatura realizada ofrece unas conclusiones generales que establecen, con claridad, el carácter coste-efectivo de la mayor parte de las intervenciones analizadas. Una extrapolación lógica de estos datos permite afirmar que la puesta en práctica de un modelo de atención basado en este tipo de intervenciones en la CAPV sería potencialmente costeefectiva. En ese sentido, cabe pensar que el desarrollo de este tipo de intervenciones permitiría no sólo mantener o mejorar la situación de las personas atendidas en términos de calidad de vida, mejora del estatus funcional y de salud, autodeterminación, integración o satisfacción con los servicios prestados, sino, además, hacerlo destinando a esos programas un nivel de recursos económicos igual o inferior.

Junto a esta conclusión general, los resultados más significativos de la revisión realizada son los siguientes:

- Se han detectado cinco tipos de intervenciones - la gestión de casos, las ayudas tecnológicas y para la adaptación de la vivienda, los programas de autocuidado, las fórmulas de alojamiento en la comunidad y la atención intermedia- que: a) han sido sometidas a un número elevado de evaluaciones, y b) resultan mayoritariamente costeefectivas. Desde ese punto de vista, y aun teniendo en cuanta las limitaciones señaladas en el epígrafe anterior, cabe pensar que cualquier modelo de atención comunitaria e individualización de los cuidados que quiera desarrollarse en nuestro entorno deberá incorporar, en la mayor medida posible, este tipo de intervenciones.

- Existe otro grupo de intervenciones -los servicios sociosanitarios integrados, la ayuda a domicilio y los programas de individualización de la atención-que también resultan mayoritariamente coste-efectivos, si bien el número de evaluaciones revisadas es sustancialmente menor, y la base para sostener su coste-efectividad es, por tanto, más limitada.

- Por último, hay un número relativamente elevado de intervenciones -los programas de prevención de caídas, los servicios de día, las visitas domiciliarias preventivas, los servicios de respiro, las asignaciones económicas individuales, los servicios de rehabilitación, los servicios de recapacitación a domicilio o los servicios de acompañamiento- que no han podido demostrar su coste-efectividad.

- En la mayor parte de estos casos, sin embargo, el número de evaluaciones revisadas es reducido, por lo que su carácter no coste-efectivo no puede establecerse con rotundidad. Además, cabe señalar que en pocos casos los programas se consideran no coste-efectivos (es decir, no consiguen resultados mejores al mismo o inferior coste); por lo general, estos estudios arrojan resultados no concluyentes o muestran una relación coste-efectividad no dominante (en la que, generalmente, se obtienen mejores resultados a costes superiores, o el gasto realizado es mayor que el ahorro inducido).
- No puede decirse, por otra parte, que la metodología empleada implique un sesgo importante en los resultados. Los estudios que han utilizado una metodología a priori más exigente, los estudios controlados aleatorios, resultan coste-efectivos en el $67 \%$ de los casos, y los estudios controlados no aleatorios, en el $66 \%$. Ambos tipos de estudios se encuentran, de hecho, entre los que registran un porcentaje más elevado de resultados coste-efectivos.

- El relativamente elevado número de investigaciones no concluyentes pone de manifiesto la necesidad de intensificar los esfuerzos dirigidos a analizar la relación coste-efectividad de los programas, sobre todo en aquellos casos en los que los resultados parecen menos claros (servicios de respiro, de atención diurna, prestaciones económicas). En ese sentido, la revisión realizada en este informe puede contribuir al diseño, en la CAPV, de un modelo de evaluación económica que permita establecer, con el necesario rigor, la relación coste-efectividad de los diversos servicios y prestaciones.

Con esta base, los elementos que cabe subrayar para la definición de un modelo de atención en la CAPV son los siguientes:

- La coordinación de los servicios sociales y sanitarios se ha mostrado altamente coste-efectiva, especialmente cuando se consigue la integración total de la atención para determinado colectivo. Aún en los casos en los que la completa integración no resulta posible por limitaciones organizativas, uno de sus elementos clave, la gestión de casos, sí resulta coste-efectiva incluso cuando se aplica de forma aislada. Esta forma de trabajo supone crear equipos multidisciplinares y designar a un gestor de casos, que adoptará la responsabilidad de coordinar y hacer un adecuado seguimiento de toda la atención que requiere la persona mayor. Este enfoque coincide, precisamente, con el adoptado en la iniciativa experimental Etxean Ondo (〈http://www.ingema. es/es/projects/etxean-ondo/>), desarrollada por la Fundación Matia.

- La promoción de la salud de las personas mayores por medio de programas de autocuidado también resulta una intervención preventiva altamente coste-efectiva que se podría implantar en el modelo que la Fundación Matia está desarrollando. Estas intervenciones, cuando están bien insertadas en la rutina de la persona mayor e implican la realización de actividades significativas relacionadas con la vida diaria, pueden mejorar el estado de salud de los mayores y suponer un importante ahorro para los servicios sanitarios y sociales.

- Las fórmulas de alojamiento residencial para personas mayores deben estar bien insertadas en su comunidad, permitiendo la participación de las personas en la mayor medida posible. Aunque 
no puede garantizar la calidad de la atención, ésta se ve favorecida en entornos pequeños con un ambiente hogareño. La investigación llevada a cabo en el ámbito de la discapacidad pone de manifiesto, en cualquier caso, que las 'deseconomías de escala' sólo se producen a partir de tamaños muy reducidos, inferiores a tres plazas.

- La adecuada prestación de ayudas técnicas y pequeñas adaptaciones en la vivienda puede suponer un importante ahorro de recursos, en la medida en que previene la utilización de servicios más costosos, tal y como han puesto de manifiesto los estudios revisados. En este sentido, un programa de adaptaciones mínimas complementario al servicio de ayuda a domicilio -ayudas técnicas para facilitar la entrada y salida de la bañera (alfombra, asideros y tabla de transferencia), sentarse y levantarse del retrete (elevador de retrete y asideros) y de la butaca (alzas), el desplazamiento en el interior de la casa (bastón) y entrada y salida de la cama (alzacamas y barandilla)- podría suponer un importantísimo ahorro para la Administración si se proveyera de forma sistemática a todas las personas usuarias del servicio de ayuda a domicilio.

- Los servicios de respuesta rápida y de intervención en crisis, dirigidos a personas mayores y con discapacidad en riesgo de ser admitidas en un hospital o un centro residencial (por un accidente o emergencia) pueden liberar camas hospitalarias y prevenir ingresos residenciales definitivos, lo que supone un importante ahorro para la Administración.

- De acuerdo con los resultados de esta revisión, los servicios de respiro deberían limitarse, de momento, al respiro residencial, el único que ha demostrado algún grado de coste-efectividad. Por otra parte, hay que tener en cuenta que, en el caso de personas mayores cuidadas por sus cónyuges, los servicios de respiro residencial, más que prevenir el ingreso definitivo, pueden provocarlo o precipitarlo. Sí se consideran una intervención coste-efectiva y de carácter preventivo en el caso de personas mayores que son cuidadas por sus hijos/as.

- La recapacitación a domicilio, a pesar de que su coste-efectividad todavía no se ha podido demostrar de forma concluyente, tiene muchas posibilidades de resultarlo, por su capacidad de reducir las necesidades de ayuda formal de las personas que pasan por el servicio, por lo que se recomienda su adopción en la CAPV, al menos de forma experimental. 
AH-SOUNE, M. F.; y DE VIGNERTE, B. (2000): "Intérêts et couts d'une réinsertion gériatrique après séjour en soins de suite et réadaptation", La Revue de Geriatrie, vol. 25, no 7 , págs. 471-476.

ANDRICH, R.; y CARACCIOLO, A. (2007): "Analysing the cost of individual assistive technology programmes", Disability and Rehabilitation: Assistive Technology, vol. 2, nํㅜ 4, págs. 207-234.

ARKSEY, H. et al. (2004): Review of Respite Services and Short-Term Breaks for Carers for People with Dementia, Londres, NHS Service Delivery and Organisation R\&D Program.

ARTASO, B. et al. (2002): "Análisis coste-consecuencia de un centro de día psicogeriátrico", Revista Española de Geriatría y Gerontología, vol. 37, nำ6, págs. 291-297.

ASHTON, T.; HEMPENSTALL, C. (2009): Research into the Financial Benefits of the Supporting People Programme, 2009, Londres, Department for Communities and Local Government.

BAUMKER, T. et al. (2008): Costs and Outcomes of an ExtraCare Housing Scheme in Bradford, York, Joseph Rowntree Foundation.

BÈLAND, F. et al. (2006): “A system of integrated care for older persons with disabilities in Canada: Results from a randomized controlled trial", The Journals of Gerontology, vol. 61A, no 4 , págs. 367-373.

BENDIXEN, R. M. et al. (2009): “Cost effectiveness of a telerehabilitation program to support chronically ill and disabled elders in their homes", Telemedicine Journal and E-Health, vol. 15, nํㅜ 1, págs. 31-38.

BOUMAN, A. et al. (2008): "Effects on health care use and associated cost of a home visiting program for older people with poor health status: A randomized clinical trial in the Netherlands", The Journals of Gerontology, vol. 63A, $n \div 3$, págs. 291-297.

CHALLIS, D. et al. (2004): “The value of specialist clinical assessment of older people prior to entry to care homes", Age and Ageing, vol. 33, no 1 , págs. 25-34.

CHAPPELL, N. L. et al. (2004): “Comparative costs of home care and residential care", The Gerontologist, vol. $44, n^{0} 3$, págs. 389-400.

CHARLESWORTH, G. et al. (2008): Does Befriending by Trained Lay Workers Improve Psychological Well-Being and Quality of Life for Carers of People with Dementia, and at What Cost? A Randomised Controlled Trial, serie Health Technology Assessment Report, no 4, Southampton, NIHR Coordinating Centre for Health Technology Assessment.

CLARKSON, P. et al. (2010): “Targeting, care management and preventative services for older people: The cost effectiveness of a pilot self-assessment approach in one local authority", British Journal of Social Work, vol. 40, nํ- 7, págs. 2.255-2.273.

COLEMAN, E. A. et al. (2006): "The care transitions intervention. Results of a randomized controlled trial", Archives of Internal Medicine, vol. 166, págs. 1.822-1.828.

COUNSELL, S. R. et al. (2009): "Cost analysis of the geriatric resources for assessment and care of elders care management intervention", Journal of the American Geriatrics Society, vol. 57, nํㅛ 8, págs. 1.420-1.426.

CUNLIFFE, A. L. et al. (2004): "Sooner and healthier: A randomised controlled trial and interview study of an early discharge rehabilitation service for 
older people", Age and Ageing, vol. 33, n- 3 , págs. 246-252.

CURRY, N. (2006): Preventive Social Care. Is It Cost Effective?, Londres, King's Fund.

DALE, S. B.; y BROWN, R. (2006): “Reducing nursing home use through consumer-directed personal care services", Medical Care, vol. 44, no 8, págs. 760-767.

DAVIS, J. C. et al. (2010): "Does a home-based strength and balance programme in people aged $=$ 80 years provide the best value for money to prevent falls? A systematic review of economic evaluations of falls prevention interventions", British Journal of Sports Medicine, vol. 44, $\mathrm{n}-2$, págs. 80-89.

ELLIS, A. et al. (2006): "Buying time II: An economic evaluation of a joint $\mathrm{NHS} /$ social services residential rehabilitation unit for older people on discharge from hospital", Health and Social Care in the Community, vol. $14, \mathrm{n}-2$, págs. 95-106.

EMERSON, E. et al. (2005): "Costs and outcomes of community residential supports in England", en STANCLIFFE, R. J.; y LAKIN, K. C. (eds.), Costs and Outcomes of Community Services for People with Intellectual Disabilities, Baltimore, Paul H. Brookes, págs. 151-174.

FELCE, D.; y EMERSON, E. (2005): “Community living. Costs, outcomes, and economies of scale: Findings from U.K. research", en STANCLIFFE, R. J.; y LAKIN, K. C. (eds.), Costs and Outcomes of Community Services for People with Intellectual Disabilities, Baltimore, Paul H. Brookes, págs. 45-62.

FELCE, D. et al. (2008): "Outcomes and costs of community living: Semi independent living and fully staffed group homes", American Journal on Mental Retardation, vol. 113, nํㅜ 2, págs. 87-101.

FRICK, K. D. et al. (2010): "Evaluating the cost effectiveness of fall prevention programs that reduce fall related hip fractures in older adults", Journal of the American Geriatrics Society, vol. 58, $\mathrm{n}^{0}$ 1, págs. 136-141.

GAUGLER, J. E. et al. (2003): “Evaluating community-based programs for dementia caregivers: The cost implications of adult day services", Journal of Applied Gerontology, vol. 22, № 1, págs. 118-133.

GRABOWSKI, D. C. (2006): "The cost-effectiveness of noninstitutional long-term care services: Review and synthesis of the most recent evidence", Medical Care Research and Review, vol. $63, \mathrm{n}-1$, págs. 3-28.

HAY, J. et al. (2002): "Cost-effectiveness of preventive occupational therapy for independent-living older adults", Journal of the American Geriatrics Society, vol. 50, no 8 , págs. 1.381-1.388.

HEAD, M. J.; y CONROY, J. W. (2005): "Outcomes of selfdetermination in Michigan. Quality and costs", en STANCLIFFE, R. J.; y LAKIN, K. C. (eds.), Costs and Outcomes of Community Services for People with Intellectual Disabilities, Baltimore, Paul H. Brookes, págs. 219-240.

HEBERT, R. et al. (2008): "Les impacts du réseau intégré de services Prisma”, Gérontologie et Société, no 124 , págs. 15-47.
HEKTOEN, L. F. et al. (2009): "Cost-effectiveness in fall prevention for older women”, Scandinavian Journal of Public Health, vol. 37, n으, págs. 584-589.

HEYWOOD, F.; y TURNER L. (2007): Better Outcomes, Lower Cost. Implications for Health and Social Care Budgets of Investment in Housing Adaptations, Improvements and Equipment: A Review of the Evidence, Londres, HM Government.

HURSTFIELD, J. et al. (2007): The Costs and Benefits of Independent Living, Londres, HM Government.

IRVINE, L. et al. (2010): “Cost effectiveness of a day hospital falls prevention programme for screened community dwelling older people at high risk of falls", Age and Ageing, vol. 39, no 6, págs. 710-716.

JOHRI, M. et al. (2003): "International experiments in integrated care for the elderly: A synthesis of the evidence", International Journal of Geriatric Psychiatry, vol. 18, no 3, págs. 222-235.

KAAMBWA, B. et al. (2008): "Costs and health outcomes of intermediate care: Results from five UK case study sites", Health and Social Care in the Community, vol. 16, no 6, págs. 573-581.

KENNEDY, A. et al. (2007): "The effectiveness and cost effectiveness of a national lay-led self care support programme for patients with long-term conditions: A pragmatic randomised controlled trial", Journal of Epidemiology and Community Health, vol. 61, nํㅜ 3, págs. 254-261.

LAKIN, K. C. et al. (2005): "Home and community-based services. Costs, utilization, and outcomes", en STANCLIFFE, R. J.; y LAKIN, K. C. (eds.), Costs and Outcomes of Community Services for People with Intellectual Disabilities, Baltimore, Paul H. Brookes, págs. 91-127.

LANSLEY, P. et al. (2004): “Can adapting the homes of older people and providing assistive technology pay its way?", Age and Ageing, vol. $33, \mathrm{n} \div$ 6, págs. 571-576.

LEUNT, A. C. et al. (2004): "Cost-benefit analysis of a case management project for the communitydwelling frail elderly in Hong Kong", Journal of Applied Gerontology, vol. 23, $\mathrm{n}^{0}{ }_{1}$, págs. 170-185.

MANSELL, J.; y BEADLE-BROWN, J. (2008): Dispersed or Clustered. Housing for Disabled Adults: $A$ Systematic Review, serie Disability Research Series, no 12, Dublín, National Disability Authority.

MANSELL, J. et al. (2007): Deinstitutionalisation and Community Living. Outcomes and Costs: Report of a European Study. Volume 2: Main Report, Canterbury, Tizard Centre.

MARKLE-REID, M. et al. (2006): "Health promotion for frail older home care clients", Journal of Advanced Nursing, vol. 54, nº 3, págs. 381-395.

MARKLE-REID, M. et al. (2006): "The effectiveness and efficiency of home-based nursing health promotion for older people: A review of the literature", Medical Care Research and Review, vol. 63, nº 5, págs. 531-569.

MASON, A. et al. (2007): A Systematic Review of the Effectiveness and Cost-Effectiveness of Different 
Models of Community-Based Respite Care for Frail Older People and Their Carers, serie Health Technology Assessment Report, $\mathrm{n} 0 \mathbf{1 5}$, Southampton, NIHR Coordinating Centre for Health Technology Assessment.

MCLEOD, B.; y MAIR, M. (2009): Evaluation of City of Edinburgh Council Home Care Re-ablement Service, Edimburgo, Scottish Government Social Research.

MELIS, R. J. F. et al. (2008): “Cost-effectiveness of a multidisciplinary intervention model for community-dwelling frail older people", The Journals of Gerontology, vol. 63A, nํㅜ 3 , págs. $275-282$

MEYER, H. (2011): "A new care paradigm slashes hospital use and nursing home stays for the elderly and the physically and mentally disabled", Health Affairs, vol. 30, noํ 3, págs. 412-415.

MILLER, P. et al. (2005): “Economic analysis of an early discharge rehabilitation service for older people", Age and Ageing, vol. 34, n으 3, págs. 274-280.

NICHOLS, L. O. et al. (2008): "The cost-effectiveness of a behavior intervention with caregivers of patients with Alzheimer's disease", Journal of the American Geriatrics Society, vol. 56, no 3 , págs. 413-420.

O’REILLY, J. et al. (2008): “Post-acute care for older people in community hospitals. A cost-effectiveness analysis within a multi-centre randomised controlled trial", Age and Ageing, vol. 37, $\mathrm{n}^{\circ}$ 5, págs. 513-520.

PICKARD, L. (2003): The Effectiveness and Cost-Effectiveness of Support and Services to Informal Carers of Older People. A Review of the Literature Prepared for the Audit Commission, Londres, Audit Commission.

POVEDA, R. et al. (2009): Análisis de costes y beneficios de la incorporación de productos de apoyo como extensión de los servicios de atención a domicilio en población mayor con dependencia. Ponencia presentada en el I Congreso de la Red Española de Política Social, Oviedo, noviembre 2009.

RICHARDS, K. C. et al. (2005): "Effect of individualized social activity on sleep in nursing home residents with dementia", Journal of the American Geriatrics Society, vol. 53, no 9, págs. 1.510-1.517.

RICHARDSON, G. et al. (2008): “Cost effectiveness of the Expert Patients Programme (EPP) for patients with chronic conditions", Journal of Epidemiology and Community Health, vol. 62, n- 4, págs. 361-367.

ROBERTSON, J. et al. (2008): “Análisis longitudinal del impacto y coste de la planificación centrada en la persona para personas con discapacidad intelectual en Inglaterra", Siglo Cero, vol. 39, $\mathrm{n} 01,225$, págs. 5-30.

ROBERTSON, J. et al. (2004): "Quality and costs of community-based residential supports for people with mental retardation and challenging behavior", American Journal on Mental Retardation, vol. 109, nํㅜ 4, págs. 332-344.

ROBERTSON, M. C. et al. (2001): "Economic evaluation of a community based exercise programme to prevent falls", Journal of Epidemiology and

Community Health, vol. 55, no 8, págs. 600-606.

SAHLEN, K. G. et al. (2008): "Preventive home visits to older people are cost-effective", Scandinavian Journal of Public Health, vol. 36, no 3 , págs. 265-271.

SCHWAB, T. C. et al. (2003): "Home- and community-based alternatives to nursing homes: Services and costs to maintain nursing home eligible individuals at home", Journal of Aging and Health, vol. 15, nํㅡ 2, págs. 353-370.

SPREAT, S. et al. (2005): "A cost-benefit analysis of community and institutional placements for persons with mental retardation in Oklahoma", Research in Developmental Disabilities, vol. 26, no 1 , págs. 17-31.

STAINTON, T. et al. (2009): “Independence pays: A cost and resource analysis of direct payments in two local authorities", Disability and Society, vol. 24, no 2 , págs. 161-172.

STANCLIFFE, R. J.; y KEANE, S. (2000): "Outcomes and costs of community living: A matched comparison of group homes and semi-independent living", Journal of Intellectual and Developmental Disability, vol. 25, nํ4, págs. 281-305.

STANCLIFFE, R. J.; y LAKIN, C. (2004): "Costs and outcomes of community services for persons with intellectual and developmental disabilities", Policy Research Brief, vol. 15, nํㅜ 1 .

TIMONEN, V. (2004): Evaluation of Homecare Grant Schemes in the NAHB and ECAHB, Dublín, Eastern Regional Health Authority.

TOSELAND, R. W.; y SMITH, T. L. (2006): “The impact of a caregiver health education program on health care costs", Research on Social Work Practice, vol. 16, nํㅜ 1, págs. 9-19.

TOUSIGNANT, M. et al. (2003): "Economic evaluation of a geriatric day hospital: Cost-benefit analysis based on functional autonomy changes", Age and Ageing, vol. 32, nํㅜ 1, págs. 53-59.

TURNING POINT (2010): Benefits Realisation: Assessing the Evidence for the Cost Benefit and Cost Effectiveness of Integrated Health and Social Care, Londres, Turning Point.

VV.AA. (2000): The Way to Go Home: Rehabilitation and Remedial Services for Older People, serie Promoting Independence, $\mathrm{n}-\mathbf{4}$, Londres, Audit Commission.

WAGNER, L. M. et al. (2007): “Description of an advanced practice nursing consultative model to reduce restrictive side-rail use in nursing homes", Research in Nursing and Health, vol. 30, $\mathrm{n}^{\circ} \mathbf{2}$, págs. 131-140.

WATT, S. et al. (1999): “Community care for people with chronic conditions: An analysis of nine studies of health and social service utilization in Ontario", The Milbank Quarterly, vol. 77, nํㅜ 3, págs. 363-392.

WINDLE, K. et al. (2009): National Evaluation of Partnerships for Older People Projects. Final Report, Kent, Personal Social Services Research Unit. 